\title{
Joint Channel Estimation and Data Detection with Cubature Kalman Filters
}

\author{
Raquel G. Machado, Luis G. P. Meloni and Renato R. Lopes
}

\begin{abstract}
Resumo-Este artigo apresenta a utilização do cubature Kalman filter para a estimação dos coeficientes de um canal MIMO duplamente seletivo e detecção de símbolos discretos de forma conjunta. Com base no modelamento da dinâmica temporal dos coeficientes do canal e dos símbolos transmitidos, um vetor de estados é definido contendo ambas informações levando a uma não-lineraridade na equação de observação do sistema proposto. $\mathrm{O}$ cubature Kalman filter é então utilizado para estimar as variáveis de estado, acompanhando satisfatoriamente a variação temporal do canal e reconhecendo os símbolos transmitidos com baixa taxa de erros.
\end{abstract}

Palavras-Chave-Estimação e Detecção Conjuntas, Canal Duplamente Seletivo, Solução em Espaço de Estados, Filtro de Curvatura de Kalman.

Abstract-In this paper we approach the problem of joint channel estimation and data detection in multiple-input multipleoutput (MIMO) systems subject to fast frequency-selective fading using the cubature Kalman filter (CKF). First, based on a model of the channel coefficients and transmitted symbol dynamics, the problem is formulated in the state-space form, leading to a nonlinear observation equation. Once the filtering problem is defined, the CKF is presented and its numerical implementation is discussed. Finally, through numerical simulations, it is shown that the CKF can be used to track channel variations and detect transmitted symbols with low error rates.

Keywords-Joint Estimation and Detection, Fast FrequencySelective Fading, State-Space Approach, Cubature Kalman Filter.

\section{INTRODUCTION}

The use of multiple transmission and/or reception antennas [1], [2], has played a central role in the design of modern wireless communication systems. In practice, when using high data transmission rates in these systems, the transmitted symbols are subject to inter-symbol interference (ISI). Furthermore, if there exists a relative movement between the receiver and the transmitter, the wireless channel through which data is transmitted will vary in time, becoming a fast frequencyselective channel. Therefore, in order to implement practical wireless systems that use multiple transmit and/or receive antennas, it is essential to devise methods to correctly detect symbols transmitted under such conditions.

Also, in order to mitigate channel effects, the receiver must employ estimators for channel tracking and equalizers

Raquel G. Machado, Luis G. P. Meloni and Renato R. Lopes are with the Department of Communications of the School of Electrical and Computer Engineering, University of Campinas, C.P. 6101, CEP 13081-970, Campinas, SP, Brazil, \{raquel, meloni, rlopes \} @decom. fee.unicamp.br

We acknowledge the financial support received from FINEP (6341) and CNPq (311844/2006-5) for ISI compensation. Usually, the estimators and equalizers work separately, and, in this type of scheme, the correlation between estimated channel and data symbols introduced by using estimates from detection in channel estimation process and vice-versa is not considered. An interesting alternative to this usual approach is described in [3], where a joint semiblind detection and channel estimation is proposed using the Bayesian estimation theory.

In Bayesian filtering [4], [5], the posterior density of the state of a system is used to evaluate the time and measurement updates. The filter evaluates the statistical expectation of these conditional densities through multi-dimensional integrals, providing an optimal analytical solution to non-linear filtering problems. However, the solution of these integrals involves great computational cost resulting in a mathematical problem. Therefore, approximations to the theoretical Bayesian filter are necessary in order to obtain practical non-linear filters. In this sense, a common solution for non-linear filtering problems is provided by the extended Kalman filter (EKF) [6], which is based on local linear approximations to the Bayesian filter using the first order Taylor series. However, there is a wide variety of scenarios where this first order approximation does not suffice, leading to a large estimation error and thus limiting the applicability of the EKF.

In order to overcome the limitations of presently known filters, the cubature Kalman filter (CKF), presented in [7], was recently proposed. The CKF is a Gaussian approximation for the Bayesian filter that provides a more accurate filtering than EKF, being able to solve a wider range of nonlinear problems. To approximate the theoretical Bayesian filter, the CKF assumes that all conditional densities are Gaussian, transforming the multi-dimensional integral into a product between a non-linear function and a Gaussian probability distribution. Also, exploiting an efficient numerical integration method known as cubature rules [8], the CKF requires little computational cost.

Within this context, our goal is to efficiently perform a joint channel estimation and data detection in multiple-input multiple-output (MIMO) systems subject to fast frequencyselective fading. In order to achieve this goal, we first model the channel estimation and data detection in the state-space form, noting that this leads to a non-linear filtering problem, and allowing us to deal with both problems simultaneously. Once the non-linear filtering problem is defined, we discuss how the cubature Kalman filter can be used to obtain measurement updates of the estimation and detection processes. Finally, we analyze the performance of the CKF for our problem through numerical simulations, comparing the results 
with the EKF, which is the filter traditionally used in this type of situation [9].

The remainder of this paper is organized as follows: Section II describes the system model and the state-space formultaion. The cubature Kalman filter derivation for the considered problem is presented in Section III. Section IV reports simulation results and, finally, Section $\mathrm{V}$ concludes the paper.

\section{SySTEM MODEL}

We consider a MIMO system with $N_{T}$ transmitting antennas and $N_{R}$ receiving antennas interfering with each other. Due to ISI, a signal received by an antenna $i$ is also subject to interference from symbols transmitted previously. The relationship between received and transmitted signals at time $k$ can be expressed as:

$$
\begin{aligned}
\mathbf{y}_{k} & =\sum_{l=0}^{L-1} \mathbf{H}_{l, k} \mathbf{x}_{k-l}+\mathbf{n}_{k} \\
& =\left[\begin{array}{llll}
\mathbf{H}_{0, k} & \mathbf{H}_{1, k} & \ldots & \mathbf{H}_{L-1, k}
\end{array}\right]\left[\begin{array}{c}
\mathbf{x}_{k} \\
\mathbf{x}_{k-l} \\
\vdots \\
\mathbf{x}_{k-L+1}
\end{array}\right]+\mathbf{n}_{k}
\end{aligned}
$$

where $\mathrm{L}$ is the number of channel paths, $\mathbf{y}_{k}=$ $\left[\begin{array}{llll}y_{1, k} & y_{2, k} & \ldots & y_{N_{R}, k}\end{array}\right]^{T}$ is the vector of signals received by the $N_{R}$ antennas, $\mathbf{x}_{k}=\left[\begin{array}{llll}x_{1, k} & x_{2, k} & \ldots & x_{N_{T}, k}\end{array}\right]^{T}$ is the vector of signals transmitted by the $N_{T}$ antennas, $\mathbf{H}_{l, k}$ is a $N_{R} \times N_{T}$ matrix, $\forall l=0, \ldots, L-1$, whose element that lies on the $i-t h$ row and $j-t h$ column is denoted by $h_{i, j, l, k}$, corresponding to the value of the $l-t h$ channel coefficient between the $j-t h$ transmitting antenna and the $i-t h$ receiving antenna at time $k$, and $\mathbf{n}_{k}=\left[\begin{array}{llll}n_{1, k} & n_{2, k} & \ldots & n_{N_{R}, k}\end{array}\right]^{T}$ is a vector of white, Gaussian, circularly symmetric, zero mean, i.i.d noise samples, with variance equal to $\sigma_{n}^{2}$.

In addition, in order to represent the time variation of the MIMO channel taps, we assume the typical wide-sense stationary uncorrelated scattering (WSSUS) model [10]. In this model, the channel taps have time-autocorrelation properties that are governed by the Doppler rate and are given by

$$
E\left[h_{i, j, l, k} h_{i, j, l, k+\Delta k}^{*}\right] \approx \mathcal{J}_{0}\left(2 \pi f_{D} T_{s}|\Delta k|\right)
$$

where $\mathcal{J}_{0}$ is the zero-order Bessel function of the first kind, $f_{D} T_{s}$ is the normalized Doppler rate and $T_{s}$ is the baud duration.

We consider that the channel coefficients remain constant during $N \geq 1$ symbols and follow the time-autocorrelation function (2) for time evolution. Thus, we can stack the $N$ received vectors, and write the received signals as a linear combination of the transmitted symbols, obtaining

$$
\tilde{\mathbf{y}}_{k}=\mathcal{H} \tilde{\mathbf{x}}_{k}+\tilde{\mathbf{n}}_{k}
$$

where $\quad \tilde{\mathbf{y}}_{k}=\left[\begin{array}{llll}\mathbf{y}_{k}^{T} & \mathbf{y}_{k-1}^{T} & \ldots & \mathbf{y}_{k-N+1}^{T}\end{array}\right]^{T}$ is a column vector containing the $\mathrm{N}$ received vectors, $\tilde{\mathbf{x}}_{k}=\left[\begin{array}{llll}\mathbf{x}_{k}^{T} & \mathbf{x}_{k-1}^{T} & \ldots & \mathbf{x}_{k-N-L-2}^{T}\end{array}\right]^{T}$ is a vector containing $N+L-1$ stacked transmitted vectors,
$\tilde{\mathbf{n}}_{k}=\left[\begin{array}{llll}\mathbf{n}_{k}^{T} & \mathbf{n}_{k-1}^{T} & \ldots & \mathbf{n}_{k-N-L-2}^{T}\end{array}\right]^{T}$ is the noise vector and

$$
\mathcal{H}=\left[\begin{array}{ccccc}
\mathbf{H}_{0, k} & \ldots & \mathbf{H}_{L-1, k} & \ldots & 0 \\
0 & \mathbf{H}_{0, k} & \ldots & \mathbf{H}_{L-1, k} & 0 \\
\vdots & \ddots & \ddots & \ddots & \vdots \\
0 & 0 & \mathbf{H}_{0, k} & \ldots & \mathbf{H}_{L-1, k}
\end{array}\right]
$$

is a block Toepletiz matrix with each block representing the channel coefficients.

Also, defining $\operatorname{vec}(\cdot)$ as the operator that stacks the columns of a matrix on top of each other, the column vector

$$
\mathbf{h}_{k}=\operatorname{vec}\left(\left[\begin{array}{llll}
\mathbf{H}_{0, k} & \mathbf{H}_{1, k} & \ldots & \mathbf{H}_{L-1, k}
\end{array}\right]\right)
$$

represents the channel coefficients. As described in [14], we can approximate the channel dynamics by a first order autoregressive process (AR), and its time evolution can be written as

$$
\mathbf{h}_{k}=\beta \mathbf{h}_{k-1}+\mathbf{w}_{k}
$$

where $\beta=\mathcal{J}_{0}\left(2 \pi f_{D} T_{s}\right), \mathbf{w}_{k}$ is a vector of white, Gaussian, circularly symmetric, zero mean noise samples, with covariance matrix $\mathbf{W}=\sigma_{w}^{2} I_{N_{R} N_{T}}$, and $\sigma_{w}^{2}=\left(1-|\beta|^{2}\right)$.

Finally, (3) can be rewritten as a combination of the channel coefficients as follows:

$$
\tilde{\mathbf{y}}_{k}=\mathcal{X} \mathbf{h}_{k}+\tilde{\mathbf{n}}_{k}
$$

where

$$
\mathcal{X}=\left[\begin{array}{cccc}
\mathbf{x}_{k}^{T} & \mathbf{x}_{k-1}^{T} & \cdots & \mathbf{x}_{k-L+1}^{T} \\
\mathbf{x}_{k-1}^{T} & \mathbf{x}_{k-2}^{T} & \cdots & \mathbf{x}_{k-L}^{T} \\
\vdots & \vdots & \ldots & \vdots \\
\mathbf{x}_{k-N+1}^{T} & \mathbf{x}_{k-N}^{T} & \cdots & \mathbf{x}_{k-N-L+2}^{T}
\end{array}\right] \otimes I_{N_{R}}
$$

and $\otimes$ denotes the Kronecker product. Note that (4) and (5) suggests the formulation of a filtering problem to perform the channel tracking as in [11]. However, in a practical communication system, some elements in $\mathcal{X}$ may not be known by the receiver, preventing a linear state-space modeling.

In order to develop the joint estimation and detection state model, the augmented state vector, $\mathbf{z}_{k}$, is defined as

$$
\mathbf{z}_{k}=\left[\begin{array}{ll}
\tilde{\mathbf{x}}_{k}^{T} & \mathbf{h}_{k}^{T}
\end{array}\right]^{T} .
$$

Observe that in order to obtain the augmented state-equation, it is necessary to describe the dynamic behavior of the vector $\tilde{\mathbf{x}}_{k}$, that contains the stacked transmitted symbols. For such, note that, as time evolves, new transmitted symbols are added to the top of $\tilde{\mathbf{x}}_{k}$, while existing symbols are shifted towards the bottom. Consequently, defining $\mathbf{0}_{i \times j}$ as a $i$-by- $j$ matrix of zeros, this time-shifting structure can be modeled as

$$
\tilde{\mathbf{x}}_{k}=\mathbf{F}_{x} \tilde{\mathbf{x}}_{k-1}+\mathbf{u}_{k}
$$

where

$$
\mathbf{F}_{x}=\left[\begin{array}{cc}
\mathbf{0}_{N_{T} \times N_{T}(N+L-2)} & \mathbf{0}_{N_{T} \times N_{T}} \\
\mathbf{I}_{N_{T}(N+L-2)} & \mathbf{0}_{N_{T}(N+L-2) \times N_{T}}
\end{array}\right]
$$

is a shift matrix and

$$
\mathbf{u}_{k}=\left[\begin{array}{ll}
\mathbf{x}_{k}^{T} & \mathbf{0}_{1 \times N_{T}(N+L-2)}
\end{array}\right]^{T}
$$


is a non-Gaussian noise with covariance matrix given by

$$
\begin{aligned}
\mathbf{U} & =E\left[\mathbf{u}_{k} \mathbf{u}_{k}^{H}\right] \\
& =\sigma_{u}^{2}\left[\begin{array}{cc}
\mathbf{I}_{N_{T}} & \mathbf{0}_{N_{T} \times N_{T}(N+L-2)} \\
\mathbf{0}_{N_{T}(N+L-2) \times N_{T}} & \mathbf{0}_{N_{T}(N+L-2) \times N_{T}(N+L-2)}
\end{array}\right]
\end{aligned}
$$

With the dynamic behavior of $\tilde{\mathbf{x}}_{k}$ and $\mathbf{h}_{k}$ in hand, (given by (7) and (4), respectively), it is possible to write the state transition matrix from time $k-1$ to time $k$ as

$$
\mathbf{F}=\left[\begin{array}{cc}
\mathbf{F}_{x} & \mathbf{0} \\
\mathbf{0} & \mathbf{F}_{h}
\end{array}\right]
$$

where $\mathbf{F}_{h}=\beta$.

Therefore, using (5)-(8), we define the process and observation equations for the problem of joint channel estimation and data detection as

$$
\begin{gathered}
\mathbf{z}_{k}=\mathbf{F z}_{k-1}+\mathbf{q}_{k} \\
\tilde{\mathbf{y}}_{k}=\mathbf{C}\left(k, \mathbf{z}_{k}\right)+\tilde{\mathbf{n}}_{k}
\end{gathered}
$$

where

$$
\mathbf{C}\left(k, \mathbf{z}_{k}\right)=\mathcal{X} \mathbf{h}_{k}=\mathcal{H} \tilde{\mathbf{x}}_{k}
$$

and

$$
\begin{aligned}
\mathbf{q}_{k} & =\left[\begin{array}{ll}
\mathbf{u}_{k}^{T} & \mathbf{w}_{k}^{T}
\end{array}\right]^{T}, \\
\mathbf{Q} & =E\left[\begin{array}{ll}
\mathbf{q}_{k} \mathbf{q}_{k}^{H}
\end{array}\right]=\left[\begin{array}{cc}
\mathbf{U} & \mathbf{0} \\
\mathbf{0} & \mathbf{W}
\end{array}\right] .
\end{aligned}
$$

Note that the process equation described in (9) is linear and allows us to estimate the predicted state $\left(\hat{\mathbf{z}}_{k \mid k-1}\right)$ and the predicted error covariance $\left(P_{k \mid k-1}\right)$ using Kalman filtering theory [9]. The observation equation, on the other hand, is a nonlinear function of the state variables, since it involves a multiplication between state vector elements. Consequently, for measurement updates, the Kalman filter can not be used and the optimal solution can only be achieved through Bayesian filtering.

\section{Cubature Kalman Filter}

The Bayesian filtering paradigm evaluates the time and measurement updates by calculating the statistical expectation of the predicted state density based on past input-measurement pairs. As discussed earlier, this requires calculating multidimensional integrals in order to obtain the predicted measurement and error covariance matrices associated with the Kalman gain. Approximating this statistical description, the CKF assumes that the conditional densities are Gaussian. Thus, defining $a_{k \mid k-1}$ as the estimate of a term $a$ at time $k$ given observation up to $k-1$, the innovation covariance matrix, the cross covariance matrix and the predicted measurement are as follows

$$
\begin{gathered}
P_{y y, k \mid k-1}=\int_{\mathcal{R}^{n z}}\left(\mathcal{H}_{k \mid k-1} \tilde{\mathbf{x}}_{k \mid k-1}\right)\left(\mathcal{H}_{k \mid k-1} \tilde{\mathbf{x}}_{k \mid k-1}\right)^{T} \\
* \mathcal{N}\left(\mathbf{z}_{k} ; \hat{\mathbf{z}}_{k \mid k-1}, \mathbf{P}_{k \mid k-1}\right) d \mathbf{z}_{k} \\
-\hat{\mathbf{y}}_{k \mid k-1} \hat{\mathbf{y}}_{k \mid k-1}^{T}+\sigma_{n}^{2} \mathbf{I}_{N_{R}}, \\
P_{z y, k \mid k-1}=\int_{\mathcal{R}^{n z}} \mathbf{z}_{k}\left(\mathcal{H}_{k \mid k-1} \tilde{\mathbf{x}}_{k \mid k-1}\right)^{T}
\end{gathered}
$$

$$
\begin{gathered}
* \mathcal{N}\left(\mathbf{z}_{k} ; \hat{\mathbf{z}}_{k \mid k-1}, \mathbf{P}_{k \mid k-1}\right) d \mathbf{z}_{k} \\
-\hat{\mathbf{z}}_{k \mid k-1} \hat{\mathbf{y}}_{k \mid k-1}^{T}
\end{gathered}
$$

and

$\hat{\mathbf{y}}_{k \mid k-1}=\int_{\mathcal{R}^{n z}} \mathcal{H}_{k \mid k-1} \tilde{\mathbf{x}}_{k \mid k-1} * \mathcal{N}\left(\mathbf{z}_{k} ; \hat{\mathbf{z}}_{k \mid k-1}, \mathbf{P}_{k \mid k-1}\right) d \mathbf{z}_{k}$

where

$$
\hat{\tilde{\mathbf{x}}}_{k \mid k-1}=\left[\begin{array}{ll}
\mathbf{0}_{1 \times N_{T}}^{T} & \left.\hat{\tilde{\mathbf{x}}}_{k-1 \mid k-1}\left(1: N_{T}(N+L+2)\right)^{T}\right]
\end{array}\right.
$$

and

$$
\mathcal{H}_{k \mid k-1}=\beta\left[\begin{array}{ccccc}
\mathbf{H}_{0, k} & \ldots & \mathbf{H}_{L-1, k} & \ldots & 0 \\
0 & \mathbf{H}_{0, k} & \ldots & \mathbf{H}_{L-1, k} & 0 \\
\vdots & \ddots & \ddots & \ddots & \vdots \\
0 & 0 & \mathbf{H}_{0, k} & \ldots & \mathbf{H}_{L-1, k}
\end{array}\right]
$$

As mentioned before, an analytic solution for these integrals is mathematical intractable. However, in order to numerically calculate such integrals, a third degree spherical-radial rule, called cubature rule [7], can be used. According to the cubature rule, a standard Gaussian weighted integral can be computed as follows:

$$
\int_{\mathcal{R}^{n}} \mathbf{f}(\mathbf{x}) \mathcal{N}(\mathbf{x} ; \mathbf{0}, \mathbf{I}) d \mathbf{x} \approx \sum_{i=1}^{m} \omega_{i} \mathbf{f}\left(\xi_{i}\right)
$$

where

$$
\xi_{i}=\sqrt{\frac{m}{2}}\left\{\left(\begin{array}{c}
1 \\
\vdots \\
0
\end{array}\right), \ldots,\left(\begin{array}{c}
0 \\
\vdots \\
1
\end{array}\right),\left(\begin{array}{c}
-1 \\
\vdots \\
0
\end{array}\right), \ldots,\left(\begin{array}{c}
0 \\
\vdots \\
-1
\end{array}\right)\right\}
$$

and

$$
\omega_{i}=\frac{1}{m}, i=1,2, \ldots, m=2 * \mathcal{S} .
$$

where $\mathcal{S}$ is the state dimension.

Therefore, (11), (12) and (13) can be approximated as:

$$
\begin{aligned}
& \hat{\mathbf{y}}_{k \mid k-1}=\frac{1}{m} \sum_{i=1}^{m} \mathbf{C}\left(k, S_{k \mid k-1} \xi_{i}+\hat{\mathbf{z}}_{k \mid k-1}\right) \quad, \\
& P_{y y, k \mid k-1}=\frac{1}{m} \sum_{i=1}^{m}\left[\mathbf{C}\left(k, S_{k \mid k-1} \xi_{i}+\hat{\mathbf{z}}_{k \mid k-1}\right)\right. \\
& \left.* \mathbf{C}\left(k, S_{k \mid k-1} \xi_{i}+\hat{\mathbf{z}}_{k \mid k-1}\right)^{T}\right] \\
& -\hat{\mathbf{y}}_{k \mid k-1} \hat{\mathbf{y}}_{k \mid k-1}^{T}+\sigma_{n}^{2} \mathbf{I}_{N_{R}} \text {, }
\end{aligned}
$$

and

$$
\begin{aligned}
P_{z y, k \mid k-1}= & \frac{1}{m} \sum_{i=1}^{m}\left[\left(S_{k \mid k-1} \xi_{i}+\hat{\mathbf{z}}_{k \mid k-1}\right)\right. \\
& \left.* \mathbf{C}\left(k, S_{k \mid k-1} \xi_{i}+\hat{\mathbf{z}}_{k \mid k-1}\right)^{T}\right] \\
& -\hat{\mathbf{z}}_{k \mid k-1} \hat{\mathbf{y}}_{k \mid k-1}^{T},
\end{aligned}
$$

where $S_{k \mid k-1}$ is a square-root of the predicted error covariance $P_{k \mid k-1}$.

In scenarios with high $\mathrm{SNR}$, i.e., when $\sigma_{n}^{2}$ is small, this filter may become numerically unstable. In order to mitigate such effects, we use the square-root cubature Kalman filter, which is a square-root version of the CKF. This algorithm 
TABELA I

Square-Root Cubature Kalman Filter Algorithm

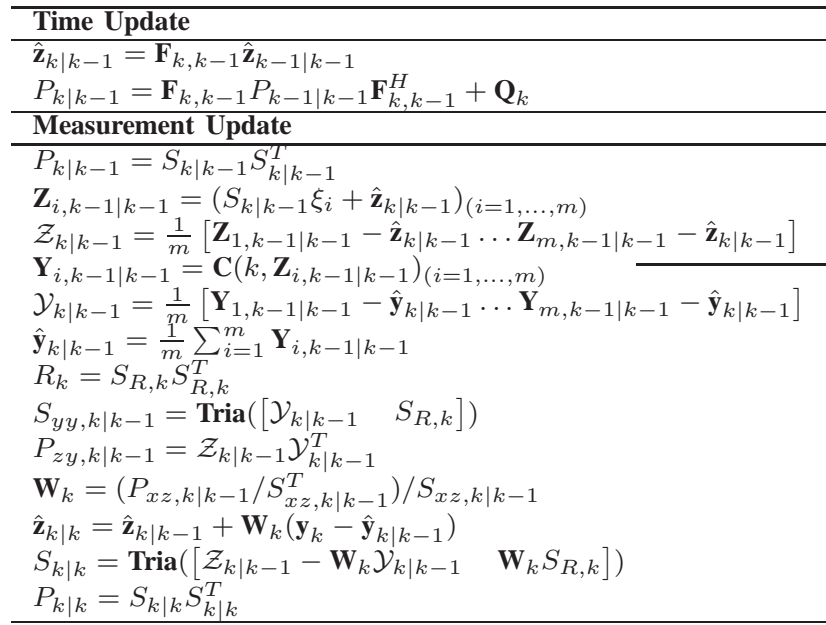

efficiently performs the joint estimation and data detection since the SCKF treats the nonlinearity inherent to the problem. Defining Tria $(X)$ as a general triangularization algorithm [12], the steps are shown in Table 1.

The use of triangularizations for covariance updates, and the use of the least-squares method for calculating the Kalman gain used in the algorithm improves the numerical stability of the SCKF. Furthermore, its computational complexity, in terms of flops, is similar to the EKF, growing proportionally to the cube of dimension of the state $z$ that is composed by the channel paths and vectors stacked for observation.

In order to correctly detect the received symbols, it is important to note that the estimated stacked vectors

$$
\hat{\tilde{\mathbf{x}}}_{k}=\left[\begin{array}{llll}
\hat{\mathbf{x}}_{k}^{T} & \hat{\mathbf{x}}_{k-1}^{T} & \ldots & \hat{\mathbf{x}}_{k-N-L-2}^{T}
\end{array}\right]^{T}
$$

contains the estimation of the current transmitted symbols vector and the estimation of vectors transmitted in past time instants. This fact suggests that a vector estimated in a later moment provides a better estimative for the transmitted symbols. Thus, a fixed delay in detection is introduced, and the final estimative for vector $\hat{\mathbf{x}}_{k}$ is obtained at time $k+N+L-2$, where a decision device gives the detected symbols.

\section{Simulations and Results}

In this section, we report the results obtained by applying the algorithm in Table 1, to the problem of joint channel estimation and data detection in MIMO wireless communication system subject to fast frequency-selective fading. We suppose a communication system as described by (3), in an environment with 2 transmitting and 4 receiving antennas, in which $1 \times 10^{6}$ QPSK symbols are sent in frames composed by 25 training symbols and 125 data symbols. The channel coefficients were generated by an autoregressive process as in (4), with $0 \mathrm{~dB}$ average power, and we assume that the receiver knows the noise variance $\sigma_{n}^{2}$.

Two scenarios were considered: a normalized Doppler rate $f_{D} T=0.001$ with $N=6$ stacked vectors and $f_{D} T=0.01$ with $N=10$. For both scenarios, the algorithm's performance

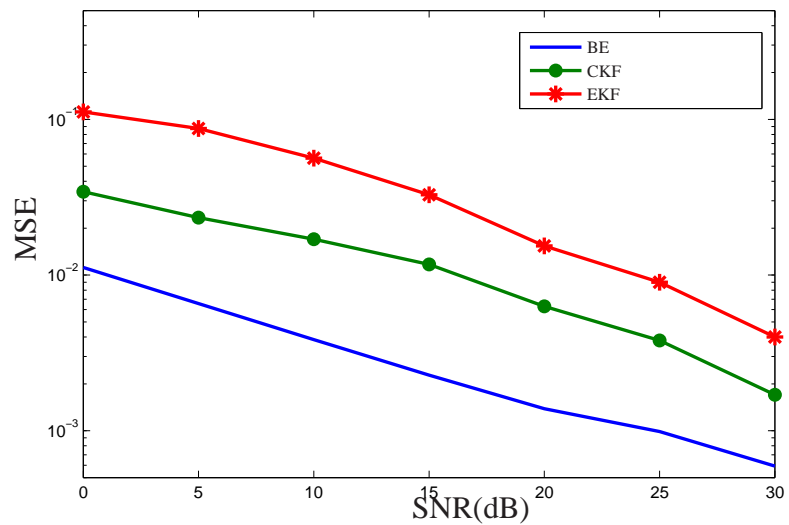

Fig. 1. Mean Square error of channel tracking for $f_{D} T=0.001$.

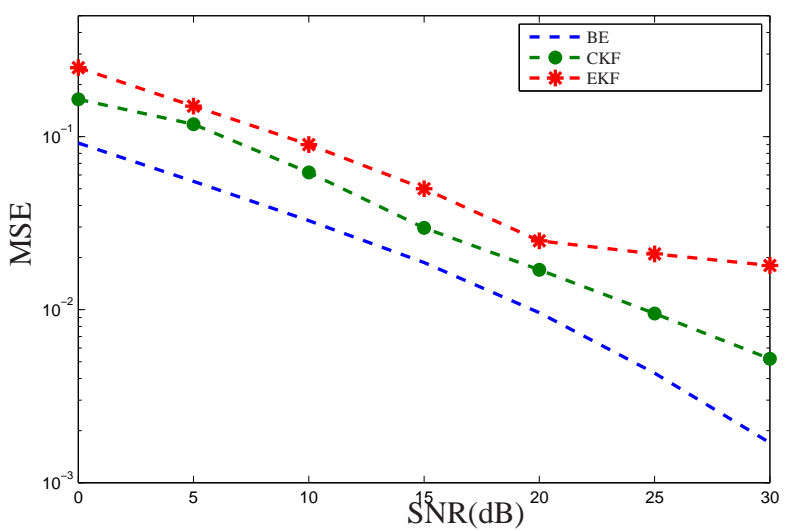

Fig. 2. Mean Square Error of channel tracking for $f_{D} T=0.01$.

can be observed from the Mean Square Error (MSE) of the channel coefficients and Symbol Error Rate (SER) analysis. The MSE and SER values for each SNR considered are compared to perfect conditions, i.e, for channel estimation, we consider the case when all symbols are known by the receiver (Best Estimation - BE) and for symbol detection, we plot the performance of a Kalman equalizer with perfect knowledge of channel state information (KF-CSI). In addition, for comparison purposes, the performance of a receiver using the extended Kalman filter (EKF) for joint estimation and detection [13] is also shown.

Fig. 1 presents the MSE of the channel estimation performed by the CKF, the EKF, and by an estimator with complete knowledge of all symbols, representing the best possible channel estimative (BE) for a normalized doppler rate of 0.001 . It is clear that the estimation provided by the CKF is better than the estimation provided by the EKF, being closer to the best possible channel estimative, which indicates the ability of the CKF in satisfactorily tracking the variation of channel coefficients. Figure 2 depicts the MSE performance of the CKF and the EKF for $f_{D} T=0.01$. Note that for higher SNR's, the EKF error performance stops improving, while the MSE values for CKF continues to decrease. This can be explained mainly by the fact that very accurate measurements 


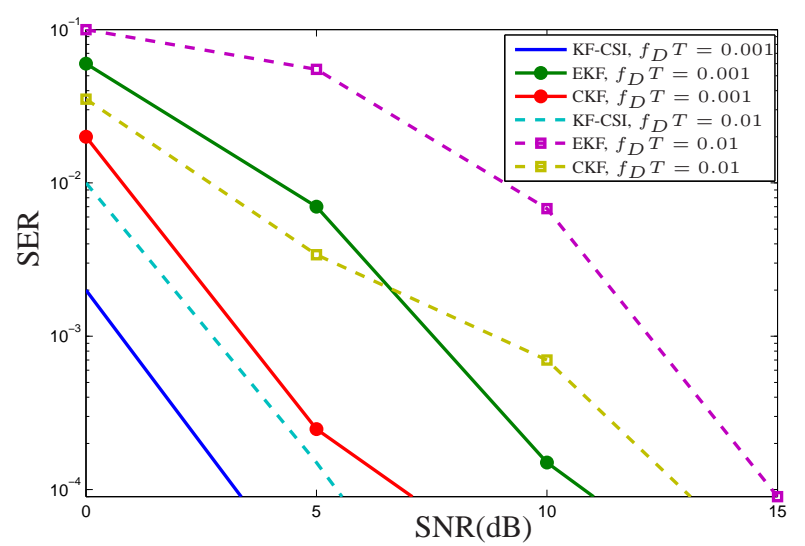

Fig. 3. Symbol error rate for detected symbols.

in some nonlinear filtering problems may result in numeric instability, which ends up interfering on channel estimation. Thus, besides being a better estimator, this result shows that the square-root cubature Kalman filter is also less prone to numerical instabilities.

The symbol error rate for detected symbols using the CKF, the EKF and a Kalman equalizer with perfect channel knowledge (KF-CSI) is presented in Fig. 3. As expected, for slower channel variations, all filters have better performances. When the normalized channel Doppler rate is 0.001 , the difference between the CKF and the KF-CSI is of approximately $3 \mathrm{~dB}$, while the difference between the EKF and the KF-CSI is of approximately $6 \mathrm{~dB}$. When we increase the frequency of channel variation to $f_{D} T=0.01$, this difference is of about $7 \mathrm{~dB}$ for the CKF and $9 \mathrm{~dB}$ for EKF. Analyzing the SER values, the CKF once again outperforms the EKF.

\section{CONCLUSIONS}

In this paper, the cubature Kalman filter (CKF), was used to perform joint channel estimation and data detection in fast frequency-selective MIMO environments. In order to jointly estimate the channel and detect the symbols, the problem was modeled in the state-space form by defining an extended state-equation containing both the channel coefficients and the transmitted symbols. This formulation leads to a nonlinear observation equation, which prevents the use of the wellknown Kalman Filter.

Once the estimation problem was defined, the cubature Kalman filter (CKF) was presented, along with its squareroot version. The square-root cubature Kalman filter (SCKF) is a variation of the traditional CKF, and was used in order to guarantee numerical stability.

Simulations proved the ability of the CKF to efficiently track fast channel variations and correctly detect symbols with a low error rate. Furthermore, the CKF outperformed the extended Kalman filter (EKF), presenting a smaller MSE for channel estimation and a smaller SER for symbol detection than the EKF, which is the commonly nonlinear filtering solution. The simulations also show that the CKF is less susceptible to numerical issues than the EKF, despite the fact that both algorithms have comparable computational complexities.

\section{REFERÊNCIAS}

[1] E. Biglieri, R. Calderbank, A. Constantinides, A. Goldsmith, A. Paulraj and H. Vincent Poor, MIMO Wireless Communications, Cambridge University Press, 2007.

[2] D. Tse and P. Viswanath, Fundamentals of Wireless Communication, Cambridge University Press, May 2005.

[3] R. Piechocki, A. Nix, J. McGeehan, and S. Armour, "Joint blind and semi-blind detection and channel estimation," IEEE Proceedings on Communications, vol. 150, 2003, pp. 419-426.

[4] Y. Ho and R. Lee, "A Bayesian approach to problems in stochastic estimation and control," IEEE Transactions on Automatic Control, vol. 9, pp. 333-339, 1964.

[5] V. Peterka, "Bayesian Approach To System Identification,"in Proc. in Trends and Progress in System Identification, P. Eykhoff, Ed, vol. 1, 1981, pp. 239-304.

[6] T. Kaylath, A. H. Sayed, e B.Hassibi, Linear Estimation, Prentice Hall, 2000.

[7] I. Arasaratnam, and S. Haykin, "Cubature Kalman Filters," IEEE Trans. Automatic Control, To Appear in Vol. 54, No 6, Jun., 2009.

[8] R.Cools, "Constructing cubature formulae: the Science behind the art, "Acta Numerica 6, pp 1-54, 1997.

[9] D. Schafhuber, G. Matz, and F. Hlawatsch, "Kalman tracking of timevarying channels in wireless MIMO-OFDM systems,"in Proc. Asilomar, 2003, vol.2, pp. 1261-1265.

[10] W.C. Jakes, Microwave Mobile Communications, New York: John Wiley and Sons, 1974.

[11] M. Enescu, T. Roman, and V. Koivunen, "State-space approach to spatially correlated MIMO OFDM channel estimation,"Signal Processing, vol. 87 , Set. 2007, pp. 2272-2279.

[12] E. Anderson, Z. Bai, C. Bischof, S. Blackford, J. Demmel, J. Dongarra, J.Du Croz, A. Greenbaum, S. Hammarling, A. McKenney, and D. Sorensen, LAPACK User's Guide, Third Edition, SIAM, Philadelphia, 1999.

[13] M. Loiola and R. Lopes, "A state-space approach to semi-blind signal detection in fast frequency-selective fading MIMO channels, "in Proc. IEEE 9th Workshop on Signal Processing Advances in Wireless Communications (SPAWC), 2008, pp. 276-280.

[14] C. Komninakis, C. Fragouli, A. Sayed, and R. Wesel, "Multi-input multi-output fading channel tracking and equalization using Kalman estimation,"IEEE Transactions on Signal Processing, vol. 50, 2002, pp. 1065-1076. 